\title{
The Necessary Existence of Gravitational Waves and the Repulsive Gravitation
}

\author{
C. Y. Lo \\ Applied and Pure Research Institute, 15 Walnut Hill Rd., Amherst, NH 03031, USA
}

\begin{abstract}
It is exciting that the gravitational wave has been confirmed, according to the announcement of LIGO. Perhaps, this is the time for physicists to fix the Einstein equation for the gravitational wave and the nonexistence of the dynamic solution. These two problems are inextricably related. As a first step, theorists should improve their pure mathematics beyond Einstein on non-linear mathematics and related physical considerations. Then, theoretically we must first show that the gravitational waves necessarily exist that Einstein was not certain. We find that the existence of gravitational waves is due to that the photons must have a gravitational wave component. Next, we must rectify the Einstein equation that has no gravitational wave solution which Einstein has recognized, and no dynamic solution that Einstein failed to see. However, it is very questionable that the measured gravitational waves are due to the black holes that can be definitely valid due to the long distance of the sources. Moreover, since the repulsive gravitation can also generate a gravitational wave, the problem of gravitational wave is actually far more complicated than we have known. A useful feature of the gravitational wave based on repulsive gravitation is that it can be easily generated on earth. Thus this can be a useful tool for communication because it can penetrate any medium.
\end{abstract}

Key words: Anti-gravity coupling, gravitational radiation, principle of causality.

\section{Introduction}

The LIGO announced that gravitational wave has been detected [1]. Congratulations to those who worked hard for this achievement. In the announcement, it is claimed that Einstein's prediction on the gravitational wave has been fulfilled. However, this is not an accurate story of Einstein on this subject. Although, based on the linearization of his equation, Einstein had predicted the existence of gravitational waves, and later he was also the first to discover that his non-linear field equation has no gravitational wave solution $[2,3]$.

In fact, Einstein concluded his talk on gravitational waves at Princeton University by saying [4] "If you ask me whether there are gravitational waves or not, I must answer that I do not know. But it is a highly interesting problem." Thus, Einstein's last words on this subject were that "I do not know." 1) Obviously,

Corresponding author: C. Y. Lo, Ph. D. in mathematics from Queen's University, D. Sc. in physics from MIT, research field: theoretical physics.
Einstein was puzzled by why his linearized equation predicted the existence of the gravitational solutions, but his non-linear Einstein equation rejects such a claim. Now, it is time to rectify the Einstein equation.

The reason of Einstein's puzzle is due to his inadequacy in mathematics that he did not see that linearized Einstein equation and the non-linear Einstein equation actually are independent equations for the dynamic case although for the static case these two equations are compatible. Since the linearized equation has not been justified, the existence of gravitational waves was not certain. ${ }^{2)}$ In this paper, it will be shown that the existence of gravitational waves is intimately related to the notion of photons.

Moreover, in general relativity mass is not the only source of gravitation, ${ }^{3)}$ since it has been shown that the electromagnetic energy can be the source of the repulsive gravitation [5]. In this paper, we shall discuss also the gravitational wave generated by electromagnetic energy and its applications. 


\section{The Conflict between the Einstein Equation and the Formula $\mathrm{E}=\mathbf{m c}^{2}$}

We usually cite the achievements of Einstein on his field equation and the formula $\mathrm{E}=\mathrm{mc}^{2}$. However, few recognized that they are actually not always consistent [6]. Since the Einstein equation is the basis of the numerous predictions the only choice seems to be just what can be done on the unverified formula $\mathrm{E}=\mathrm{mc}^{2}$ [7].

From general relativity $[8,9]$, the Einstein equation is

$$
\mathrm{G}_{\mu \nu} \equiv \mathrm{R}_{\mu \nu}-\frac{1}{2} \mathrm{~g}_{\mu \nu} \mathrm{R}=-\kappa \mathrm{T}_{\mu \nu}
$$

where $\mathrm{g}_{\mu v}$ is the space-time metric, $\mathrm{R}_{\mu \nu}$ is the Ricci curvature tensor, $R=R_{\alpha \beta} g^{\alpha \beta}$ is the Ricci curvature, $T_{\mu \nu}$ is the sum of energy-stress tensors and $\kappa$ is the coupling constant. According to the Einstein Eq. (1), we have

$$
\mathrm{R}=\mathrm{R}_{\alpha \beta} \mathrm{g}^{\alpha \beta}=\kappa \mathrm{T}_{\alpha \beta} \mathrm{g}^{\alpha \beta}
$$

Since the electromagnetic energy-stress tensor is traceless, the electromagnetic energy cannot affect the Ricci curvature R, but the mass can. Thus, it is clear that the electromagnetic energy cannot be equivalent to mass. $^{4)}$

One might argue that experiments show that a $\pi_{0}$ meson can decay into two photons. This would support that the electromagnetic energy is equivalent to mass, according to the proposal of Einstein [10] that photons consist of only electromagnetic energy. However, experimentally, Einstein's proposal is only partially verified since nobody has verified that the photons consist of only pure electromagnetic energy. In view of the fact that all the charged particles are massive, it is entirely possible that the photons would include gravitational energy. Thus, we must investigate the gravity of an electromagnetic wave to see whether the gravitational wave exists.

\section{Inadequacy of Einstein's Notion of Photons and Invalidity of $\mathrm{E}=\mathbf{m c}^{2}$}

It is the bending of light ray that makes Einstein famous. Here, it will be shown that the bending of light also exposed the shortcomings of Einstein. Moreover, the electromagnetic energy must generate different gravitation [11].

In Einstein's calculation, it is implicitly assumed that the gravity created by an electromagnetic wave is negligible. Since Einstein also claimed that any energy-momentum tensor can be a source of his equation, one should be able to obtain a gravitational solution for the electromagnetic wave. Since such gravity is very weak in physics, journals such as the Chinese Physics B and Physical Review D, in agreement with Einstein, believed that such gravity can be calculated with the perturbation approach, but they did not do it.

Mathematically, for a perturbation approach to be valid, a necessary condition is, however, that this problem has a bounded solution. This compatibility between mathematics and physics is crucial for the validity of a theory in physics. Thus, it is natural for Einstein [12] to believe that his equation could be used for such a case.

For a plane electromagnetic wave, the metric obtained by Penrose [13] is the following:

$$
d s^{2}=d u d v+H d u^{2}-d x_{i} d x_{i}
$$

where $u=c t-z, v=c t+z$

$$
H=h_{i j}(u) x_{i} x_{j}, \quad \mathrm{~h}_{\mathrm{ij}}(\mathrm{u}) \text { is the energy-momentun }
$$
tensor.

Since the solution (3) is not bounded, this violates the principle of causality and also common sense. Moreover, since there are non-physical parameters (the choice of origin) that are unrelated to any physical causes, 5) this violates the principle of causality further. However, according to the standard of the Physical Review [14] the solution of Penrose was perfectly valid. This shows that the editors of APS have a deficiency in physics.

Penrose is essentially a mathematician, and thus causality was probably absent from his training, but this lack of awareness in causality from the editors for the Review of Modern Physics, should be a surprise. 
Moreover, explicit calculation based on causality shows that it is impossible to have a bounded solution for the gravity of an electromagnetic wave. In order for Einstein's theory to make sense, the related Einstein equation with an electromagnetic wave as the source, must include a photonic energy-stress tensor with the anti-gravity coupling $[15,16]$.

For this case the related modified Einstein equation is the following:

$$
\mathrm{G}_{\mathrm{ab}} \equiv \mathrm{R}_{\mathrm{ab}}-\frac{1}{2} \mathrm{~g}_{\mathrm{ab}} \mathrm{R}=-\mathrm{K}\left[\mathrm{T}(\mathrm{E})_{\mathrm{ab}}-\mathrm{T}(\mathrm{p})_{\mathrm{ab}}\right]
$$

and

$$
\mathrm{T}_{\mathrm{ab}}=-\mathrm{T}(\mathrm{g})_{\mathrm{ab}}=\mathrm{T}(\mathrm{E})_{\mathrm{ab}}-\mathrm{T}(\mathrm{P})_{\mathrm{ab}}
$$

where $T(E)_{a b}$ and $T(P)_{a b}$ are respectively the energy-stress tensors for the electromagnetic wave and the related photons. Thus the photonic energy must include also the energy of its gravitational wave component.

Therefore, Einstein also failed to see the need of an anti-gravity coupling in this case, in addition to the case of a massive dynamic Einstein equation [17]. Note that it is this failure to see the need of the anti-gravity coupling that provides the erroneous theoretical basis for the space-time singularity theorems of Hawking and Penrose which actually are irrelevant to physics [18].

If the photons consist of only electromagnetic energy, there is a conflict since the photonic energy can be equivalent to mass and the electromagnetic energy is not. Now, this conflict is resolved since the photonic energy is the sum of electro-magnetic energy and gravitational energy, and thus it is established that $\mathrm{E}=\mathrm{mc}^{2}$ can be invalid.

Note that both quantum theory and relativity are based on the phenomena of light. It is gravity that makes the notion of photons compatible with the electromagnetic wave. Since Einstein proposed the photons before he conceived general relativity; understandably he failed to include gravitational wave energy. Now, since a charged particle is always massive, it would be natural to include the gravitational wave energy in a photon. Ohanian [19] incorrectly credited von Laue for complete proof of the equivalence of mass and photonic energy. However, the fact is that both von Laue and Einstein failed [20].

\section{The Dynamic Solution for the Einstein Equation and the Principle of Causality}

Because of inadequacy in non-linear mathematics, Einstein and his followers fail to see the non-existence of a dynamic solution for the Einstein equation. ${ }^{6)}$ This error led to accepting the positive mass theorem of Yau and Schoen $[21,22]$ as generally valid, ${ }^{7)}$ and thus delayed the development of general relativity for at least 13 years [23].

The issue on the existence of a dynamic solution started with the perihelion of Mercury. In 1915 Einstein obtained the expected value of the remaining perihelion with his equation, and thus was confident on his theory. His confidence was subsequently boosted by the confirmation of the bending of light [8]. However, his equation was questioned by Gullstrand [24], the Chairman (1922-1929) of the Nobel Prize for Physics. The perihelion of Mercury is actually a many-body problem [11], but Einstein had not shown that his calculation could be derived from such a step. Thus, D. Hilbert, who approved Einstein's calculation, did not come to its defense [17].

Consequently, Einstein was awarded a Nobel Prize by virtue of his photo-electric effects ${ }^{8)}$ instead of general relativity as commonly expected [17]. The fact is, however, Gullstrand is right. In 1995, it was proven that Einstein's equation is incompatible with gravitational radiation and does not have a dynamic solution [25-27].

For space-time metric $g_{\mu v}$, for massive sources, the Einstein equation of $1915[8,9]$ is

$$
G_{\mu \nu} \equiv R_{\mu \nu}-\frac{1}{2} g_{\mu \nu} R=-\kappa T(m)_{\mu \nu}
$$

where $T(m)_{\mu \nu}$ is the energy-stress tensor for massive matter.Eq. (5) implies no gravitational waves to carry 
away energy-momentum in vacuum and thus the principle of causality ${ }^{9)}$ is violated. This is the physical reason that there is no dynamic solution for the Einstein equation. Thus, Einstein's theory is at least incomplete.

There are serious consequences from the mistaken existence of dynamic solutions for the Einstein equation. A well-known result is the existence of the so-called space-time singularities due to implicitly assuming the unique sign for all the coupling constants [28]. Another result is that the misleading [29] positive mass theorem of Yau and Schoen [21, 22]. Moreover, the unification of gravitation and electromagnetism was not recognized as necessary by others [11]. Thus, the criticism of Gullstrand turns out to be very crucially constructive and beneficial.

Nevertheless, there were erroneous claims for the existence of a dynamic solution because of inadequacy in non-linear mathematics among physicists. Mathematicians such as Yau [21] and Witten [22] supported the existence of a bounded dynamic solution with their misleadingly positive mass theorem [29]. ${ }^{10)}$ Moreover, Christodoulou and Klainerman [30] invalidly claimed they have constructed dynamic solutions [31]. Not only were their errors accepted by the 1993 Nobel Prize Committee for Physics [32] but also Christodoulou was awarded the 2011 Shaw Prize ${ }^{11)}$ in mathematics [33] in honor of his errors against the honorable Gullstrand. Subsequently, Christodoulou was accepted as a member of U.S. National Academy of Sciences. Thus, generations of physicists are misled into serious errors because of the over confidence. This is highlighted by a big error, the award of the APS Medal for Exceptional Achievement in Research to Witten without experimental supports [34].

Moreover, Gullstrand was not the only theorist who questioned the existence of the bounded dynamic solution for the Einstein equation. As shown by Fock [35], any attempt to extend the Maxwell-Newton approximation to higher approximations leads to divergent terms. An independent evidence for the absence of a bounded dynamic solution is, as shown by $\mathrm{Hu}$, Zhang and Ting [36], that a gravitational radiation would depend on the approach used.

Nevertheless, many believed that the non-linear Einstein equation must have a bounded dynamic solution since the linearized Einstein equation has bounded dynamic solutions. Einstein and his peers failed to see that for a dynamic case, they are actually independent equations [6]. Thus the recent awards and honors to Christodoulou manifest an unpleasant fact that most of the theorists do not understand non-linear mathematics adequately.

\section{Gravitational Waves and the Lorentz-Levi-Einstein Equation}

Einstein incorrectly assumed that linearization was always valid to obtain an approximate solution for the nonlinear field equation [6]. However, these facts are absent from the announcement, and if it is not intended omissions, this would indicate that those working on the gravitational waves, do not fully understand Einstein's theory. They probably also do not know that the linearized equation is a valid linearization only of the Lorentz-Levy-Einstein equation [11],

$$
\mathrm{G}_{\mu \nu} \equiv \mathrm{R}_{\mu \nu}-\frac{1}{2} \mathrm{~g}_{\mu \nu} \mathrm{R}=-\mathrm{K}\left[\mathrm{T}(\mathrm{m})_{\mu \nu}-\mathrm{t}(\mathrm{g})_{\mu v}\right]
$$

where $\mathrm{t}(\mathrm{g})_{\mu \nu}$ is the energy-stress tensors for gravity and is of the first order in $\mathrm{K}^{12)}$ However, the linearized equation is actually unrelated to the Einstein equation [6], which has no bounded dynamic solution [25-27].

From Eq. (6), it is clear the physical reason that the Einstein equation does not have a dynamic solution is due a violation of the principle of causality [25], when $\mathrm{t}(\mathrm{g})_{\mu \nu}$ is absent from the source. Moreover, although Einstein realized that his equation does not have the gravitational wave solution, he did not see that the problem of the gravitational wave and the non-existence of the dynamic solution of his equation 
are inextricably related $[6,11]$.

Historically, Einstein and Rosen [2] could be considered as the first to discover the non-existence of wave solutions, but editors of the Physical Review found that the singularities they discovered are removable [14]. The editors of the Physical Review accepted unbounded solutions as valid, ${ }^{13)}$ and thus led to a self-deceptive satisfaction that hindered progress in physics [11]. The editors of journals such as the Physical Review D, the Royal Society Proceedings A and etc. did not recognize this violation of physical principles because they did not understand the principle of causality [25]. Moreover, due to elementary errors in mathematics [31] 14), Christodoulou and Klainerman [30] claimed to have constructed dynamic solutions for the Einstein equation.

A root of these problems is due to that physical space-time coordinates are ambiguous in Einstein's theory. Such an ambiguity is a consequence of Einstein's theoretical errors on measurements [37, 38]. The correct criticisms of Whitehead [39] and Zhou [40] were not accepted since they have not provided a solid theoretical foundation for general relativity to explain impressive observational confirmations. Although the physical meaning of the space-time coordinates has been clarified recently [37, 38], the existing conceptual problems still seem to grasp many theorists.

15) Fortunately, the analysis on plane-waves initiated by Liu and Zhou [41, 42] would give a simple illustration of the non-existence of the wave solution.

Currently many physicists, including Einstein, regarded gravitation is simply a branch of Riemannian geometry. This is simply incorrect. Since there are gravitational radiations in general relativity, the radiation reaction force must be included. However, there is no radiation reaction force from the geodesic equation, the equation of motion of general relativity $[8,11]$. Thus, it is clear that general relativity needs a fundamental rectification.
Another serious mistake of Einstein is his speculation of $\mathrm{E}=\mathrm{mc}^{2}$ as unconditionally valid [43] although he failed to prove it [7]. This leads to overlooking the repulsive gravitation generated by non-massive sources.

\section{The Repulsive Gravitation Generated by Electromagnetism}

The repulsive gravitation should have been discovered long time ago from the metric of a charged particle. It should have been discovered as early as 1916. From a solution of the static Einstein equation for a charged particle $\mathrm{Q}$, with mass $\mathrm{M}$ and charge q, the Reissner-Nordstrom metric [44] is as follows:

$d s^{2}=\left(1-\frac{2 M}{r}+\frac{q^{2}}{r^{2}}\right) d t^{2}-\left(1-\frac{2 M}{r}+\frac{q^{2}}{r^{2}}\right)^{-1} d r^{2}-r^{2} d \Omega^{2}$

(with $\mathrm{c}=1$ ) where $\mathrm{r}$ is the radial distance (in terms of the Euclidean-like structure [37]) from the particle center. In metric (7), the gravitational components generated by electricity have not only a different radial coordinate dependence but also a different sign that makes it a new repulsive gravitation in general relativity.

For a single charged particle, the term $\mathrm{q}^{2} / \mathrm{r}^{2}$ is very small. Nevertheless, a similar term $\mathrm{Q}^{2} / \mathrm{R}^{2}$ will be considerably large for a metal ball with sufficient charge $\mathrm{Q}$, where $\mathrm{R}$ is the distance from the center of the ball [45].

However, owing to the belief that the electric energy had a mass equivalence, ${ }^{16)}$ theorists including Einstein, and t' Hooft [46] consider the mass $M$ would include the electric energy. ${ }^{17)}$ Then, the net effect is that there would be no repulsive gravitation from a charged ball. Nevertheless, Tsipenyuk and Andreev [47] observed a weight reduction of a charged metal ball. Thus, the existence of repulsive gravitation is confirmed by experiments. 


\section{The Charge-Mass Repulsive Force and Unification}

Another problem for the existence of the repulsive gravitation in the Reissner-Nordstrom metric is that it makes clear that general relativity is incomplete. To show the static repulsive effect of a charged particle, one needs to consider only $g_{t t}$ in metric (7). According to Einstein [8], the equation of motion is the geodesic equation. For a test particle $P$ with mass $\mathrm{m}$ at $r$, the force on $P$ is

$$
\left(-m \frac{M}{r^{2}}+m \frac{q^{2}}{r^{3}}\right) \hat{r}
$$

where $\hat{r}$ is a unit vector, in the first order approximation because $g^{r r} \cong-1$. (One need not worry whether the gauge is physically valid because the gauge affects only the second order approximation of $g_{t t}[48]$.) Thus, the second term is a repulsive force.

If the particles are at rest, then the force generated by $p$ acting on the charged particle $Q$ would be

$$
\left(m \frac{M}{r^{2}}-m \frac{q^{2}}{r^{3}}\right) \hat{r}
$$

where $\hat{r}$ is a unit vector because the action and reaction forces are equal and in the opposite directions. However, for the motion of the charged particle with mass $\mathrm{M}$, if one calculates the metric according to the particle $P$ of mass $\mathrm{m}$, only the first term is obtained. Thus, the geodesic equation is inadequate to be the equation of motion.

It is necessary to have a repulsive force with the coupling $q^{2}$ to the particle $Q$ in a gravitational field generated by masses. Thus, force (9) to particle $Q$ is beyond the theoretical framework of gravitation + electromagnetism. In other words, as predicted by Lo, Goldstein, and Napier [49], general relativity leads to a realization of the inadequacy of general relativity, just as electricity and magnetism lead to their shortcomings.

The charge-mass repulsive force $m q^{2} / r^{3}$ for two point-like particles is inversely proportional to the cube power (instead of the square) of the distances between the two particles. This would mean that such a repulsive force would diminish faster than gravity at long distance. ${ }^{18)}$ Moreover, this force is proportional to the square of the charge $\mathrm{q}$, and thus is independent of the charge sign. These would make the repulsive effects verifiable [50].

The term of repulsive force in metric (2) comes from the electric energy [43]. An immediate question would be whether such a charge-mass repulsive force $m q^{2} / r^{3}$ is subjected to electromagnetic screening. It is conjectured that this force, being independent of a charge sign, would not be subjected to such a screening although it would be in general relativity. Also, this force can be considered as a result of a field created by the mass $m$ and the field interacts with the $q^{2}$ (see next section). Thus such a field is independent of the electromagnetic field.

\section{Extension of Einstein's Equivalence Principle and the Five-Dimensional Relativity}

If we consider the coupling with $\mathrm{q}^{2}$, this naturally leads to a theory of five-dimensional space of Lo, Goldstein and Napier [49]. However, the five-dimensional theory of Kaluza [51] and Einstein and Pauli [52] are excluded because they do not have the coupling with the square of a charge since the "extra" metric elements are neglected

One may ask what the physical meaning of the fifth dimension is. The position of Lo. Goldstein and Napier [49] is that the physical meaning of the fifth dimension is not yet very clear, except some physical meaning is given in the equation, $\mathrm{dx}^{5} / \mathrm{d} \tau=\mathrm{q} / \mathrm{Mc}^{2} \mathrm{~K}$ where $\mathrm{M}$ and $\mathrm{q}$ are respectively the mass and charge of a test particle, and $K$ is a constant. This equation relates the fifth variable $\mathrm{x}^{5}$ to $\tau$.

The fifth dimension is assumed [49] as part of the physical reality, and the metric signature is $(+,-,-,-$, - ). We shall denote the fifth axis as the w-axis (w stands for "wunderbar", in memorial of Kaluza), and 
thus the coordinates are $(\mathrm{t}, \mathrm{w}, \mathrm{x}, \mathrm{y}, \mathrm{z})$. Our approach is to find out the full meaning of the w-axis as our understanding gets deeper.

For a static case, we have the forces on the charged particle $Q$ in the $\rho$-direction ( $\rho$ starts from the center of p)

$$
\begin{aligned}
-\frac{m M}{\rho^{2}} & \approx \frac{M c^{2}}{2} \frac{\partial g_{t t}}{\partial \rho} \frac{d c t}{d \tau} \frac{d c t}{d \tau} g^{\rho \rho}, \\
\text { and } \frac{m q^{2}}{\rho^{3}} & \approx-\Gamma_{\rho, 55} \frac{1}{K^{2}} \frac{q^{2}}{M c^{2}} g^{\rho \rho}
\end{aligned}
$$

and

$$
\begin{gathered}
\Gamma_{k, 55} \frac{q}{K M c^{2}} \frac{d x^{k}}{d \tau}=0, \\
\text { where } \Gamma_{k, 55} \equiv \frac{\partial g_{k 5}}{\partial x^{5}}-\frac{1}{2} \frac{\partial g_{55}}{\partial x^{k}}=-\frac{1}{2} \frac{\partial g_{55}}{\partial x^{k}}
\end{gathered}
$$

in the (-r)-direction. The meaning of $(10 \mathrm{~b})$ is the energy momentum conservation. It is interesting that the same force would come from a different type of metric element depending on the test particle used. Thus,

$$
g_{t t}=1-\frac{2 m}{\rho c^{2}}, \text { and } g_{55}=\frac{m M c^{2}}{\rho^{2}} K^{2}+\text { constant }(11)
$$

In other words, $g_{55}$ is a repulsive potential. Because $g_{55}$ depends on $M$, it is a function of local property, and this is different from the metric element $g_{t t}$ that depends on a distant source of mass $\mathrm{m}$.

Thus, this force, though acting on a charged particle, would penetrate electromagnetic screening. From the above, it is possible that a charge-mass repulsive potential would exist for a metric based on the mass $M$ of the charged particle $Q$. However, because $P$ is neutral, there is no charge-mass repulsion force on $P$.

That the repulsive gravitational potential can be generated from a mass, would explain the fact that a charged capacitor can also have the repulsive force [50], but such a force is absent ${ }^{19)}$ from the current four-dimensional theory. This is why many theorists would not accept the existence of the repulsive gravitation.

\section{The Charge-Mass Interaction and the Reduction of Weight}

It is found that a charge may generate a gravitational static field that repulses a mass [43]. Since the discovery and the prediction are based on general relativity, Einstein's theory would have another important confirmation to be verified [43]. Thus, there is a new neutral charge-mass interaction that is beyond electromagnetism and gravitation, and thus as shown Einstein's unification is a necessity [43].

Moreover, since this new force is independent of the charge sign, it should not be subjected to electromagnetic screening. Nevertheless, such a coupling exists in the five-dimensional relativity of Lo, Goldstein and Napier [49]. In addition, their theory would support that such a neutral force is not subjected to electromagnetic screening. It thus follows that the existence of this static neutral repulsive force can be tested by weighing a capacitor to see whether its weight is reduced after being charged [5]. Although the charge-mass interaction is very weak, its collective effect can be measured [45]. The existence of such a force on a capacitor was first verified by Liu [5] ${ }^{20)}$ although the weight reduction of charged capacitors has been found much earlier [53]. Thus, it is found that a weight reduction of a neutral object may not be due to a reduction of mass, but a neutral repulsive force, which was unknown to Einstein [54].

Some physicists considered the weight reduction is due to a reduction of mass $[55,56]$. However, one can show experimentally the mass is still essentially the same. This can be done by measuring the period of a pendulum [54]. The period will not change if the mass is reduced. However, the period will be extended if the weight is reduced.

\section{The Current-Mass Interaction and the Repulsive Gravitational Wave}

If the electric energy leads to a repulsive force toward a mass, according to general relativity, the 
magnetic energy would lead to an attractive force from a current toward a mass [20]. The existence of such a current-mass attractive force has been verified by Martin Tajmar and Clovis de Matos [57] from the European Space Agency. They found that a spinning ring of superconducting material increases its weight much more than expected. Thus, they incorrectly believed that general relativity had been proven wrong. However, according to quantum theory, spinning superconductors should produce a weak magnetic field. Thus, they are also measuring the interaction between an electric current and the earth.

The existence of the current-mass attractive force would solve a puzzle, i.e., why a charged capacitor exhibits the charge-mass repulsive force since a charged capacitor has no additional electric charges? In a normal situation, the charge-mass repulsive force would be cancelled by other forms of the current-mass force as Galileo, Newton and Einstein implicitly assumed. This general force is related to the static charge-mass repulsive force in a way similar to the

Lorentz force is related to the Coulomb force.

One may ask what the formula for the current-mass force is. However, unlike the static charge-mass repulsive force, which can be derived from general relativity, this general force would be beyond general relativity since a current-mass interaction would involve acceleration of charges, this force would be time-dependent and generates electromagnetic radiation. Moreover, when the radiation is involved, the radiation reaction force and the variable of the fifth dimension must be considered [49]. Thus, we are not ready to derive the current-mass interaction.

Nevertheless, we may assume that, for a charged capacitor, the resulting force is the interaction of net macroscopic charges with the mass. Experimentally, we have found that the weight reduction is proportional to the square of the potential difference in the capacitor. Thus, we can generate a repulsive gravitational wave by applying an alternative electric potential to a capacitor. Since a gravitational wave can penetrate any medium, such a wave would be suitable for communication purpose.

\section{Weight Reduction by Heat}

This current-mass interaction also explains a phenomenon, which is verified by Liu [5] that it takes time for a capacitor to recover its weight after being discharged. A discharged capacitor needs time to dissipate the heat generated by discharging. Then, the motion of its electrons would recover to normal. Thus, the random moving electrons are reduced and the attractive force due to the current-mass interaction is increased.

Thus, it should be expected that the heated metals would reduce their weight [58]. It is conjectured that the heat would additionally convert some orbital electrons to random motion, but the increased mass due to heat energy is negligible as Einstein [59] pointed out. If this explanation of weight reduction is valid, then a metal would reduce its weight as the temperature increases. This should be further tested experimentally.

Moreover, since a heated metal is a solid, one can in principle test its mass by acceleration. (Another way to do this is to compare the periods of a pendulum before and after the metal is heated.) One can also verify the existence of the repulsive gravitation by measuring the reduction of attractive gravitation by using a torsion balance scale after the metal is heated. 21) Consider the repulsive gravitational wave, the problem of gravitational wave is complicated.

\section{Discussions and Conclusions}

It is hoped that the confirmation of the gravitational wave would give an added impetus for recognizing the problems in theories of gravitational waves and the non-existence of dynamic solutions. Since Einstein's puzzle is due to his inadequacy in non-nonlinear mathematics, as a first step, theorists should improve their understanding in non-linear mathematics. It is also urgently needed to investigate what is the exact 
equation for the gravitational wave and the dynamic solutions because in the Lorentz-Levy-Einstein equation the exact form of the gravitational energy-stress tensor $\mathrm{t}(\mathrm{g})_{\mu \nu}$ is still not known. Since the Einstein equation is not valid for the dynamic case, there is no theoretical basis for the existence of a black hole.

Although $\mathrm{E}=\mathrm{mc}^{2}$ is well known, we still did not understand it. ${ }^{22)}$ Thus, it was very difficult to see what is wrong in this formula. A related problem in general relativity is that the errors have been developed into systematic errors because errors often covered up each other [11]. Thus unless the ultimate source of the errors has been found out with indisputable evidence, it was difficult to recognize the related errors [11].

A common mistake among those who work on the gravitational wave is that they have incorrectly believed that the linearized equation would give an approximate solution of the non-linear Einstein equation. The fact is, however, that the linearized equation and the non-linear Einstein equation are independent equations for the dynamic case. For example, in general relativity, there is no two-body bounded solution [60] although there are two-body solutions in Newtonian theory. Thus, it is incorrect to claim that general relativity has superseded Newtonian theory.

Moreover, in view of that the source of the gravitational wave is so far away, it is questionable that the physicists can determine the sources are black holes. Considering the repulsive gravitational wave, the problem of gravitational wave is far more complicated. However, many physicists are so involved in current theoretical considerations that they forget physics is based on experiments.

One might argue that the electromagnetic energy is equivalent to mass since the $\pi_{0}$ meson can decay into two photons. However, this evidence only means that the photons contain non-electromagnetic energy. In fact, general relativity implies that the photons consist of the electromagnetic energy and the gravitational energy $[15,16]$. Moreover, the formula $\mathrm{E}=\mathrm{mc}^{2}$ is inconsistent with the static Einstein equation because the electromagnetic energy-stress tensor cannot affect the Ricci curvature $\mathrm{R}$ in the Einstein equation; whereas a mass can.

Currently, the mathematics of many physicists are so poor that they are not aware that there is a problem in Einstein's theory on the gravitational wave [61]. This is due to that well-known theorists such as the Wheeler School, the Wald School, etc. all made errors in this issue [62, 63]. The Wheeler School made the invalid claim on the existence of gravitational wave solutions because they have made crucial errors in undergraduate calculus [62]. Wald has claimed the existence of the second order solutions, but has never provided one [63]. Christodoulou and Klainerman [30] actually have never completed the construction of any dynamic solutions [31].

The 2016 APS Medal for exceptional achievement in research was awarded to Edward Witten of the Institute for Advanced Study. However, upon close examination, it is only for his "discoveries in the mathematical structure of quantum physics". It is clear also that the claimed Witten's achievements have no experimental supports since it is known that both quantum gravity and string theory have no experimentally verifiable results. I was puzzled that such an award in physics actually does not have any achievement supported by experiments [64]. Thus, this is indeed an exceptional award in Galileo's standard for an achievement in physics.

Witten was graduated in history, and thus his understanding of pure mathematics is likely half-baked. Due to inadequacy in pure mathematics like many physicists such as Pauli [65], Witten also does not understand Einstein's equivalence principle (see Appendix), and thus agrees with the misinterpretation of Wheeler [45, 62]. He also does not know that the Einstein equation does not have any dynamic solution [25-27] because he believed that linearization of the Einstein equation always produces 
an approximate solution for the Einstein equation [6]. Therefore, Witten [22] adapted Yau's invalid view [21] based on the invalidly assumed existence of dynamic solutions for the Einstein equation, and proved another version of the misleading theorem on energy. However, because the mathematicians in charge do not understand physics [23], Witten was awarded the Fields Medal in 1990.

As a result, the 1993 Noble Committee changed its mind [32] and incorrectly claimed the existence of a dynamic solution and rejected Einstein's equivalence principle. Thus, we should investigate the damage done to general relativity due to Witten's errors. Because of having no experimental supports, the APS Medal award of exceptional achievement to Witten is highly inappropriate. A merit of this award is, however, exposing the shortcomings in mathematics and physics of the Selection Committee of American Physical Society. Thus, it is necessary to recover the honor of Gullstrand [24], Chairman of the Nobel Prize Committee for Physics. ${ }^{23)}$

Experimentally, an important interesting discovery is the existence of repulsive gravitation due to the charge-mass interaction. ${ }^{24)}$ Thus, a gravitational wave can also be generated from a charged capacitor which would produce the repulsive gravitation on earth. Moreover, the electromagnetic energy can also generate attractive gravitation through the current-mass interaction. Thus, the physical picture provided by Galileo, Newton and Einstein is just too simple for gravitation which involves almost everything. Also, the notion of black holes is clearly questionable since gravity is no longer always attractive, as Wheeler explicitly assumed in his simulation [20].

According to Dr. D. Kulp, there is no editor in APS (American Physical Society), who has a background in pure mathematics. In particular, Dr. Eric J. Weinberg, ${ }^{25}$ ) editor of Physical Review D, should improve his inadequacy in mathematics. Moreover, the 2016 Award of APS Medal for Exceptional
Achievement in Research was given without any experimental support, to E. Witten [66], whose errors in general relativity are known $[22,23]$. An urgent task is to find the exact equation for the wave solutions and the dynamic solutions since general relativity is incomplete [5].

\section{Acknowledgments}

This paper is dedicated to Prof. P. Morrison of MIT for unfailing guidance for over 15 years. The author wishes to express his appreciation to S. Holcombe for valuable suggestions. This publication is supported by the Chan Foundation, Hong Kong, and Szecheon Co. Hong Kong, Special Administrative Region of China.

\section{Endnotes}

1) When people claimed that Einstein believed the existence of gravitational wave, this is not the whole story. When they claimed that they have obtained a gravitational wave solution, they made the same error that P. Morrison found that the calculation of J. H. Taylor Jr. is in valid in 1996 [70].

2) The gravitational wave solution with the support of observation was claimed by J. H. Taylor Jr., who gets a Nobel Prize for the observation of the binary pulsars [32]. However, their calculation is incorrect in mathematics. Thus he could not justify his calculation when Prof. P. Morrison of MIT went to Princeton to ask for his justification [70].

3) For instance, Professor Hughes Scott of MIT did not know the existence of repulsive gravitation. Since Morrison passed away in 2005, nobody in MIT understands general relativity.

4) This argument depends on only the validity of the static Einstein equation.

5) This shows that Penrose is only a mathematician, but not a physicist.

6) Einstein failed to see the non-existence of a dynamic solution. Due to inadequacy in mathematics, he does not know that for the dynamic case, the nonlinear Einstein equation and the linearized equation are independent [6].

7) From the theorem of Witten [22], it is clear that he also does not understand general relativity.

8) Thus a Nobel Prize guarantees only partial validity of a theory.

9) The time-tested assumption that phenomena can be explained in terms of identifiable causes is called the principle of causality. This is the basis of relevance for all 
scientific investigations. This principle implies that any parameter in a physical solution must be related to some physical causes.

10) In 1993, Yau and I discussed the non-existence of a dynamic solution [71] in the Chinese University of Hong Kong. Then, Yau claimed that he has lost his earlier interest on the work of Christodoulou and Klainerman [30].

11) Some mathematicians did not take a responsible attitude toward a Prize. Members of the awarding committee often act on their faith of previous awards, but may not know the subject at all. For instance, Y.-T. Siu of Harvard University agreed to award Christodoulou a 2011 Shaw Prize, although he does not understand general relativity nor non-linear mathematics [33].

12) Historically, Eq. (6) was first proposed by Lorentz [72] and later by Levi-Civita [73] in the following form, $\kappa t(g)_{a b}=G_{a b}+\kappa T_{a b}$ such that the gravitational energy-stress tensor $t(g)_{a b}$ takes a covariant form although they have not proved its necessity. However, Einstein [12] objected to this form on the grounds that his field equation implies $t(g)_{a b}=0$. A problem of Einstein is that he has never calculated a dynamic solution explicitly and thus failed to see its nonexistence. However, the APS was not only unable to correct Einstein's error but also added some more errors to gravitation [74].

13) Thus, the editors of APS (American Physical Society) are not always correct.

14) To be exact, they forget to prove at least a bounded dynamic solution exists [31].

15) As Zhou [40] pointed out, Einstein's covariance principle assigns different physical meaning to coordinates for different gauges. Thus, it is inconsistent with his equivalence principle that gives definite measurements to the time dilation and space contractions [62]. Note that the gauge independence of light bending $[8,9]$ is only a coincidental since the same result can be obtained from unphysical gauges [75]. Moreover, a counter example was provided by Gérard and Piereaux [76]. The relation between the impact parameter $\mathrm{b}$ and the shortest distance $\mathrm{r}_{0}$ from the light ray to the center of the sun is gauge dependent [77]. Thus gauge invariance is invalid in physics. It should be noted that Einstein's covariance principle is based on an invalid application of special relativity [38].

16) Einstein had mistaken the equivalence of photonic energy and mass as equivalence of electromagnetic energy to mass [8]. The root of the problem is due to that he had proposed inadequately that the photons consist of only electromagnetic energy. Thus, the repulsive gravitation is discovered in 1997 [78].
17) This manifests that Gerard 't Hooft does not understand special relativity and Newtonian mechanics adequately.

18) Experiments show that a charged capacitor lifter would hover above earth on a certain height [53].

19) In a four dimensional theory, there is essentially no electromagnetic force outside a charged capacitor.

20) Liu used a roll-up capacitor to show that the weight reduction of a capacitor is not directional. However, earlier physicists incorrectly believed such weight reduction is directional $[5,53]$.

21) The experiment based on the torsion balance scale provides a clear evidence for the repulsive gravitation [79].

22) Another Nobel Laureate Wilczek [80] also applied $m=$ $\mathrm{E} / \mathrm{c}^{2}$ without providing a justification. Since this relation in his theory is crucial for his Nobel Prize, Wilczek should work out a justification for its application.

23) A Chinese philosopher $\mathrm{Hu}$ Shih has said that in science, one can have daring assumptions, but one must also be careful in his proof. A problem of Einstein and many physicists are that they often have only the first half.

24) In the past, it was believed that gravitation was independent of the temperature $[8,9]$.

25) Eric J. Weinberg, editor of the Physical Review D, invalidly insists [7-9] that there is no difference in physics between Einstein's equivalence principle and Pauli's version [65] although Einstein pointed out that it is a misinterpretation [67]. He also accepts the unbounded solution because of inadequate understanding the principle of causality [25]. The root is that Eric J. Weinberg does not understand pure mathematics and thus failed to understand Einstein's equivalence principle as Pauli did [65]. Moreover, he does not understand not only non-linear mathematics, but also his calculus is defective. In addition, he simply does not have the wisdom to see that admitting past mistakes is the best choice for the APS as a scientific institute. Apparently, he is unaware of the 2003 work of Dmitriev et al. [55] on the weight reduction of the brass due to heat. As a result, erroneous papers on gravitation are still often published in the Physical Review D. Similarly, established journals such the Proceedings of the Royal Society, Annalen der Physik, etc. also just have too much blind faith on Einstein, and over-confidence on their out-dated knowledge in gravitation and experiments. Thus, the incompetency of Eric J. Weinberg is only the symptom of general inadequacy in mathematics of the physicists.

26) Since the 1911 assumption of Einstein [68] has been proven incorrect by experiments [8], it is beyond my comprehension that the Wheeler School [44] used it as the reference for Einstein's equivalence principle. 


\section{References}

[1] Announcement by the international LIGO collaboration of the direct detection of gravitational radiation on September 14, 2015.

[2] Einstein, A., and Rosen, N. 1937. J. Franklin Inst. 223: 43.

[3] Einstein, A. 2005. The Born Einstein Letters: Friendship, Politics, and Physics in Uncertain Times. New York: MacMillan, 122.

[4] Infeld, L. 1980. Quest: An Autobiography. New York: Chelsea.

[5] Lo, C. Y. 2012. "Gravitation, Physics, and Technology." Physics Essays 25 (4): 553-60.

[6] Lo, C. Y. 2013. "The Non-linear Einstein Equation and Conditionally Validity of Its Linearization." Intern. J. of Theo. and Math. Phys 3 (6).

[7] Einstein's Miraculous Year. 1998. Edited by John Stachel Princeton: Princeton University Press.

[8] Einstein, A., Lorentz, H. A., Minkowski, H., and Weyl, H. 1923. The Principle of Relativity. New York: Dover.

[9] Einstein, A. 1954. The Meaning of Relativity. Princeton Univ. Press.

[10] Einstein, A. 1905. "On a Heuristic Point of View Concerning the Production and Transformation of Light." Annalen der Physik 17: 132.

[11] Lo, C. Y. 2015. "Incompleteness of General Relativity, Einstein's Errors, and Related Experiments-American Physical Society March Meeting, Z23 5, 2015." J. of Advances in Physics 8 (2): 2135-47.

[12] Einstein, A.1954. "Physics and Reality (1936)" in Ideas and Opinions. New York: Crown, 311.

[13] Penrose, R. 1964. Rev. Mod. Phys. 37 (1): 215-20.

[14] Kennefick, D. 2004. "Einstein versus the Physical Review." Phys. Today (September).

[15] Lo, C. Y. 2006. "The Gravity of Photons and the Necessary Rectification of Einstein Equation." Prog. in Phys. 1: 46-51.

[16] Lo, C. Y. 2006. "Completing Einstein's Proof of E = $\mathrm{mc}^{2}$." Progress in Phys. 4: 14-8.

[17] Pais, A. 1996. Subtle is the Lord. New York: Oxford Univ. Press.

[18] Lo, C. Y. 2015. "The Question of Space-Time Singularities in General Relativity and Einstein's Errors." GJSFR 15-A (4).

[19] Ohanian, Hans. C. 2008. Einstein's Mistakes-The Human Falling of Genius. New York: Norton.

[20] Thorne, K. S. 1994. Black Holes and Time Warps. New York: Norton.

[21] Schoen and Yau, S.-T. 1981. "Proof of the Positive Mass Theorem. II." Commun. Math. Phys. 79: 231-60.

[22] Witten, E. 1981. "A New Proof of the Positive Energy
Theorem." Commun. Math. Phys. 80:381.

[23] Lo, C. Y. 2017. An Open Letter to the International Mathematics Union on the Errors in the 1982 and 1990 Fields Medal Awards." GJSFR-F 16 (6): 1-4.

[24] Gullstrand, A. 1921. Ark. Mat. Astr. Fys. 16 (8); ibid. 1922. Ark. Mat. Astr. Fys. 17 (3).

[25] Lo, C. Y. 2000. “On Incompatibility of Gravitational Radiation with the 1915 Einstein Equation." Phys. Essays 13 (4): 527-39.

[26] Lo, C. Y. 1995. "Einstein's Radiation Formula and Modifications to the Einstein Equation." Astrophysical Journal 455: 421-8. Editor S. Chandrasekhar suggests the appendix therein.

[27] Lo, C. Y. 1999. "Compatibility with Einstein's Notion of Weak Gravity: Einstein's Equivalence Principle and the Absence of Dynamic Solutions for the 1915 Einstein Equation." Phys. Essays 12 (3): 508-26.

[28] Wald, R. M. 1984. General Relativity. Chicago: The Univ. of Chicago Press.

[29] Lo, C. Y. 2014. The Errors in the Fields Medals, 1982 to S. T. Yau, and 1990 to E. Witten." GJSFR 13-F (11): 111-25.

[30] Christodoulou, D., and Klainerman, S. 1993. The Global Nonlinear Stability of the Minkowski Space. Princeton. Univ. Press.

[31] Lo, C. Y. 2000. Phys. Essays 13 (1): 109-20.

[32] The 1993 Press Release of the Nobel Prize Committee (Stockholm, 1993).

[33] Lo, C. Y. 2012. "Comments on the 2011 Shaw Prize in Mathematical Sciences-An Analysis of Collectively formed Errors in Physics." GJSFR 12-A (4).

[34] Lo, C. Y. 2016. "Comments on the 2016 Award of APS Medal for Exceptional Achievement in Research-A Big Step Backward in Physics of APS." Journal of Advances in Physics 11 (10): 4054.

[35] Fock, V. A. 1957. Rev. Mod. Phys. 29: 325.

[36] Hu, N., Zhang, D.-H., and Ding, H.-G. 1981. Acta Phys. Sinica 30 (8): 1003-10.

[37] Lo, C. Y. 2003. Chinese J. of Phys. 41 (4): 332-42.

[38] Lo, C. Y. 2005. "Misunderstandings Related to Einstein's Principle of Equivalence, and Einstein's Theoretical Errors on Measurements." Phys. Essays 18 (4): 547-60.

[39] Whitehead, A. N. 1922. The Principle of Relativity. Cambridge: Cambridge Univ. Press.

[40] Zhou (Chou), P. -Y. 1983. "On Coordinates and Coordinate Transformation in Einstein's Theory of Gravitation." In Proc. of the Third Marcel Grossmann Meetings on Gen. Relativ., 1-20.

[41] Liu, H. Y., and Zhou, P.-Y. 1985. Scientia Sincia (Series A) XXVIII (6): 628-37.

[42] Lo, C. Y. 2006. “The Gravitational 'Plane Waves' of Liu and Zhou and the Nonexistence of Dynamic Solutions for 
Einstein's Equation.” Astrophys. Space Sci. 306: 205-15.

[43] Lo, C. Y. 2012. "The Invalid Speculation of $m=E / c^{2}$, the Reissner-Nordstrom Metric, and Einstein's Unification." Phys. Essays 25 (1): 49-56.

[44] Misner, C. W., Thorne, K. S., and Wheeler, J. A. 1973. Gravitation. San Francisco: W. H. Freeman.

[45] Lo, C. Y., and Wong, C. 2006. "The Intrinsic Difference between Mass and Electromagnetic Energy and the Repulsive Effect in Gravity." Bulletin of Pure and Applied Sciences 25D (2): 109-17.

[46] 't Hooft, G. 1999. “A Confrontation with Infinity.” Nobel Lecture, December.

[47] Yu. Tsipenyuk, D., and Andreev, V. A. 2005. Physical Interpretations of the Theory of Relativity Conference. Moscow: Bauman Moscow State Technical University.

[48] Weinberg, S. 1972. Gravitation and Cosmology. New York: John Wiley Inc.

[49] Lo, C. Y., Goldstein G. R., and Napier, A. 1989. "Electromagnetic Radiation Reaction Force and Radiation Potential in General Five-Dimensional Relativity." Hadronic Journal 12 (2): 75-89.

[50] Lo, C. Y. 2015. "The Weight Reduction of Charged Capacitors, Charge-Mass Interaction, and Einstein's Unification." Journal of Advances in Physics 7 (3): 1959-69.

[51] Kaluza, Th. 1921. "Kaluza Sitzungsber, Preuss. Akad. Wiss." Phys. Math. Klasse 966.

[52] Einstein, A., and Pauli, W. 1943. Ann. Math. 44: 133.

[53] Valone, T. 2008. Electro Gravitics II. Washington DC: Integrity Research Institute.

[54] Lo, C. Y. 2011. "Could Galileo Be Wrong?" Phys. Essays 24 (4): 477-82.

[55] Dmitriev, A. L., Nikushchenko, E. M., and Snegov, V. S. 2003. "Influence of the Temperature of a Body on Its Weight." Measurement Techniques 46 (2): 115-20.

[56] Fan, L. Z., Feng, J. S., and Liu, W. Q. 2010. Engineer Sciences 8 (2): 9-11.

[57] http://news.softpedia.com/news/The-First-Test-That-Prov es-General-Theory-of-Relativity-Wrong-20259.shtml.

[58] Lo, C. Y. 2012. "On the Weight Reduction of Metals due to Temperature Increments." GJSFR 12 (7).

[59] Einstein. 1982. "' $\mathrm{E}=\mathrm{MC}^{2}$, (1946)." Ideas and Opinions. New York: Crown, 337.

[60] Lo, C. Y. 2016. "Comments on Errors of 'a Simplified Two-Body Problem in General Relativity' by S Hod and Rectification of General Relativity." $J$. of Advances in Physics 12 (1): 4188-96.

[61] Lo, C. Y. 2015. "The Development of Relativity and Einstein-The Progress of Physics Related to General
Relativity in 100 Years." J. of Advances in Physics 10 (3): 2874-85.

[62] Lo, C. Y. 2013. "Errors of the Wheeler School, the Distortions to General Relativity and the Damage to Education in MIT Open Courses in Physics." GJSFR 13 (7).

[63] Lo, C. Y. 2013. "Some Mathematical and Physical Errors of Wald on General Relativity." GJSFR 13-A (2).

[64] Lo, C. Y. 2016. "Comments on the 2016 Award of APS Medal for Exceptional Achievement in Research-A Big Step Backward in Physics of APS." Journal of Advances in Physics 11 (10): 4054.

[65] Pauli, W. 1971. Theory of Relativity. London: Pergamon Press.

[66] Lo, C. Y. 2016. "Comments on the 2016 Award of APS Medal for Exceptional Achievement in Research-A Big Step Backward in Physics of APS." Journal of Advances in Physics 11 (10): 4054.

[67] Norton, J. 1989. "What Was Einstein's Principle of Equivalence?” In Einstein's Studies Vol. 1: Einstein and the History of General of Relativity, edited by D. Howard and J. Stachel. Birkhäuser.

[68] Einstein, A. 1911. Annalen der Physik, 35.

[69] Synge, J. L. 1956. Relativity. Amsterdam: North-Holland.

[70] Lo, C. Y. 2013. "On the Nobel Prize in Physics, Controversies and Influences." GJSFR 13-A (3): 59.

[71] Lo, C. Y. 1993. "Einstein's Radiation Formula and Modifications in General Relativity." The Second William Fairbank Conference on Relativistic Gravitational Experiments, Hong Kong Polytechnic, 13-6.

[72] Lorentz, H. A. 1916. "Versl gewone Vergad Akad." Amst. 25: 468, 1380.

[73] Levi-Civita, T. 1917. R. C. Accad Lincei 26 (5): 381.

[74] "The American Physical Society and Errors in Gravitation." Presented in the APS Business Meeting at New Orleans, March 16, 2017, to be published.

[75] Lo, C. Y. 2004. "The Bending of Light Ray and Unphysical Solutions in General Relativity." Chin. Phys. (Beijing) 13 (2): 159-67.

[76] Gérard J. M., and Piereaux, S. 1999. "The Observable Light Deflection Angle.” arXiv:gr-qc/9907034 v1 8 Jul.

[77] Lo, C. Y. 2010. "On Gauge Invariance in Physics \& Einstein's Covariance Principle.” Phys. Essays 23 (3): 491.

[78] Lo, C. Y. 1997. Astrophys. J. 477: 700-4.

[79] Lo, C. Y. 2016. "The Observable Temperature Dependence of Gravitation.” Phys. Essays 29 (3): 247.

[80] Wilczek, F. 2004. "Asymptotic Freedom: From Paradox to Paradigm." Nobel Lecture (December). 


\section{Appendix: Mathematical Foundation of Einstein's Equivalence Principle}

A source of confusion is that Pauli's invalid version [65] has been mistaken as Einstein's equivalence principle although Einstein has made clear it is a misinterpretation [67]. In this Appendix, these errors are pointed out in the hope to help those who do not have an adequate pure mathematics background.

In "Gravitation" [44] of Misner, Thorne and Wheeler, there is no reference to Einstein's equivalence principle. Instead, they misleadingly refer to Einstein's invalid 1911 assumption [68] and Pauli’s invalid version [65]. ${ }^{26)}$ In addition, in their Eq. (40.14), Misner et al. [44] even failed to understand the local time of a particle at free fall.

The mathematical theorems [69] related to Einstein's equivalence principle are as follows:

Theorem 1. Given any point $P$ in any Lorentz manifold (whose metric signature is the same as a Minkowski space) there always exist coordinate systems $\left(x^{\mu}\right)$ in which $\partial g_{\mu v} / \partial x^{\lambda}=0$ at $P$.

Theorem 2. Given any time-like geodesic curve $\Gamma$ there always exists a coordinate system (the so-called Fermi coordinates) $\left(\mathrm{x}^{\mu}\right)$ in which $\partial g_{\mu v} / \partial x^{\lambda}=0$ along $\Gamma$.

In these theorems, the local space of a particle is locally constant, but not necessarily Minkowski.

However, after some algebra, a local Minkowski metric exists at any given point and along any time-like geodesic curve $\Gamma$. What Einstein added is that such a locally constant metric must be Minkowski. This is the basis of the Einstein-Minkowski condition that Einstein uses to derive the gravitational redshifts [29].

Note that, Pauli's version [65] is a simplified but corrupted version of these theorems as follows:

"For every infinitely small world region (i.e. a world region which is so small that the space- and time-

variation of gravity can be neglected in it) there always exists a coordinate system $\mathrm{K}_{0}\left(\mathrm{X}_{1}, \mathrm{X}_{2}, \mathrm{X}_{3}, \mathrm{X}_{4}\right)$ in which gravitation has no influence either in the motion of particles or any physical process."

Pauli regards the equivalence principle as merely the existence of locally constant spaces. Moreover, a local Min-kowski space at a point does not mean the existence of local Minkowski spaces at a small world region.

An error is that Pauli extended the removal of uniform gravity to the removal of gravity in a small region. This is simply incorrect in mathematics, but he does not see the difference because of inadequacy in mathematical analysis. He did not recognize that the removal of gravity in a small region, no matter how small, would be very different from a removal of gravity at one point. Apparently, neither Pauli [65] nor the Wheeler School [44] understands the mathematics of the above theorems [69]. 